\title{
Relevance of single channel signals for two-colour pyrometer process monitoring of laser powder bed fusion
}

\section{Kai Gutknecht* and Michael Cloots}

Irpd AG,

Lerchenfeldstrasse 3,

St. Gallen, CH-9014, Switzerland

Email: kai.gutknecht@irpd.ch

Email: dr.michael.cloots@irpd.ch

${ }^{*}$ Corresponding author

\section{Konrad Wegener}

Institute of Machine Tools and Manufacturing (IWF), ETH Zürich, CH-8092 Zürich, Switzerland

Email: wegener@iwf.mavt.ethz.ch

\begin{abstract}
This paper discusses the relevance of two-colour pyrometers' single channel signals for process monitoring of laser powder bed fusion metal additive manufacturing. First, examples are presented where the ratio temperature provided by the pyrometer is misleading. Following is a demonstration that such instances can be uncovered by the single channel signals, where an approach for simplified signal analysis based on the working principles of the pyrometer is provided. In closing, a validation is implemented, where the careful inspection and employment of the single channel signals yielded suitable input for a successful closed-loop process control.
\end{abstract}

Keywords: additive manufacturing; laser powder bed fusion; pyrometer; process monitoring; process control.

Reference to this paper should be made as follows: Gutknecht, K., Cloots, M. and Wegener, K. (2021) 'Relevance of single channel signals for two-colour pyrometer process monitoring of laser powder bed fusion', Int. J. Mechatronics and Manufacturing Systems, Vol. 14, No. 2, pp.111-127.

Biographical notes: Kai Gutknecht studied Mechanical Engineering at ETH Zurich and graduated with a Master's degree. At the time of his studies, he developed toolpath generating algorithms for a laser powder bed fusion lab machine. Furthermore, he investigated the application of metal additive manufacturing as a versatile and direct source of spare parts procurement for unique format parts used in the packaging industry. He was subsequently employed at Irpd AG in Switzerland in 2017 as a Mechanical Engineer for process $R \& D$ of laser powder bed fusion metal additive manufacturing. In parallel, he is a $\mathrm{PhD}$ candidate at the IWF of ETH Zurich on the topic of process monitoring and control of LPBF. 
Already during his studies at the RWTH Aachen and ETH Lausanne, Michael Cloots dealt with additive manufacturing processes during his studies in the field of mechanical engineering. After his time as a consultant for factory planning at a management consultancy for factory and production planning, as well as his time as a process engineer for transmission technology at an automotive OEM from the Munich area, he returned to university research at the end of 2010. During his PhD studies at ETH Zurich, he worked on the processability of nickel-based alloys using the AM technology LPBF. Since 2016, he has been responsible for managing the research group 'Additive Technologies' at Irpd AG.

Konrad Wegener is Full Professor and Head of the IWF (Institute of machine tools and manufacturing) at ETH Zurich since October 2003 and leads the manufacturing section of the Swiss technology transfer center inspire AG. Fields of research are today optimisation of machine tools, cyberphysical production systems, cutting technology, additive manufacturing, laser material processing and electro discharge machining. He is fellow of the CIRP. After his studies in mechanical engineering and PHD in Braunschweig 1990 he began his. industrial career at Schuler Presses GmbH \& Co. KG in Göppingen as head of design and layout planning departments. In 1999, he was charged with the general management of a newly acquired company for laser systems. Under his leadership large welding machines for shipbuilding and construction of aeroplanes, welding and cutting machines for the automotive industry and cutters for fabric have been developed and built.

\section{Introduction}

For further acceptance of laser powder bed fusion (LPBF) as an industrial metal additive manufacturing process, quality assurance and increased productivity is key (Colosimo and Grasso, 2020; Jurrens, 2013; Quarshie et al., 2012). As such, process monitoring is an important topic for increased industry approval of metal additive manufacturing due to its ability to provide in-situ quality control. Furthermore, the hereby applied sensors are a prerequisite for closed-loop process control, which promises to increase the process' efficiency and reliability. Accordingly, there is a large interest in this topic from the scientific community (Everton et al., 2016; Spears and Gold, 2016).

Amongst the huge variety of available sensors for LPBF process monitoring, as discussed by Everton et al. (2016), Lu and Wong (2018), Mani et al. (2015, 2017), Purtonen et al. (2014), Spears and Gold (2016) and Tapia and Elwany (2014), two-colour pyrometers implemented coaxially appear to be a very promising combination (Chivel and Smurov, 2011; Forien et al., 2020; Gutknecht et al., 2020; Hagedorn and Pastors, 2018; Hooper, 2018; Pavlov et al., 2010; Renken et al., 2019; Thombansen et al., 2014). This is reasoned since these sensors stand out as being relatively simple to integrate, they provide a comprehensible signal, are fairly robust and are generally industry applicable. Their output value, which is usually correlated with the melt pool surface temperature, reflects process dynamics in the $\mathrm{kHz}$ range and gives an overall and absolute value for process condition monitoring.

The downside of this sensor type lies in its lack of information density: An on-axis camera for example can provide spatial information about the melt pool, while the pyrometer normally provides one temperature, which is a scalar quantity and thus only 
holds limited information. The LPBF process however is very complex and deviations from the desired process condition might not reflect in the melt pool temperature at all, different phenomena result in the same signal change, or some parallel events cancel each other out regarding the surface temperature (Mitchell et al., 2020; Zhang et al., 2019). Additionally, the measurement principle and thus also the superiority of the two-colour pyrometer over conventional pyrometers or photodiodes is based on the grey-body assumption. Whenever this condition is violated, the so-called ratio temperature, which is being calculated based on two input channels, does not reflect the actual surface temperature (Müller and Renz, 2001). To this date, only very little literature is available assessing the fundamental principle of two-colour pyrometry when applied to LPBF (Hooper, 2018).

The research comprised in this paper addresses both downsides mentioned above in a first attempt and is based on the following hypothesis: It is advisable to collect not only the ratio temperature $\left(T_{R}\right)$ of a pyrometer, but also to consider the underlying single-channel signals ( $T_{1^{-}}$and $T_{2}$-temperature) to broaden the scope of information gathered and enable sanity checking of whether the necessary conditions for two-colour pyrometry do apply.

\section{Experimental setup}

An in-house built laboratory LPBF system equipped with two process monitoring sensors was used to conduct experiments regarding the significance of the different pyrometer channels for various process conditions. At the centre of the experiments stands an on-axis two-colour pyrometer for melt pool monitoring as the primary sensor. Secondarily employed is a high-speed camera, which is also aligned on-axis. Its purpose is to verify the thermal emissions of the melt pool spatially resolved. This chapter describes the machine in general, plus the sensors' setup and calibration. General information regarding the configuration of the build job is provided also.

\subsection{Machine and process parameters}

The centrepiece of the machine is a 3D scan head with an additional zoom axis for enlarging the beam in the focal plane. The minimal beam diameter at the working level is measured as $86 \mu \mathrm{m}\left(1 / e^{2}\right)$ and has a beam quality factor of $M^{2}<1.06$. The $1 \mathrm{~kW} \mathrm{IPG}$ fibre laser of type YLR-1000WC in use emits at a centroid wavelength of $1070 \mathrm{~nm}$. The scanner and laser are thermally stabilised at $22.5^{\circ} \mathrm{C} \pm 0.1 \mathrm{~K}$ using a water chiller. Pure nitrogen of quality 4.6 is used as inert gas to flush the hermetically sealed build chamber and maintain an oxygen content below $0.15 \%$. Stainless steel powder of type 1.4404 (316L) from Carpenter Additive was used.

This paper revolves around specimens manufactured with the process parameters provided in Table 1, typically yielding relative material densities of $99.5 \%$ and higher.

Table $1 \quad$ Standard process parameters

\begin{tabular}{ccccc}
\hline $\begin{array}{c}\text { Layer height } \\
{[\mu \mathrm{m}]}\end{array}$ & $\begin{array}{c}\text { Spot diameter } \\
1 / e^{2}[\mu \mathrm{m}]\end{array}$ & $\begin{array}{c}\text { Hatch distance } \\
{[\mu \mathrm{m}]}\end{array}$ & $\begin{array}{c}\text { Laser power } \\
c w[\mathrm{~W}]\end{array}$ & $\begin{array}{c}\text { Scan speed } \\
{[\mathrm{m} / \mathrm{s}]}\end{array}$ \\
\hline 30 & 86 & 70 & 200 & 1.2 \\
\hline
\end{tabular}


Generally, a customary cubical specimen with edge length $10 \mathrm{~mm}$ is used. Derivations of that shape are depicted in Figure 1, namely: Stair-step cubes, which are used to easily compare different process parameter deviations independently; each step (yellow) represents a distinct alteration from the standard parameters. Due to the proximity and the increased power level $(500 \mathrm{~W})$, the surrounding horse-shoe shaped part (green) inflicts spatter onto the standard cube in its centre (more details in Section 3.3). Lastly, the appended cantilever is used to investigate overhangs. These shapes were used in different build jobs to produce the data referred to below.

Figure 1 Different sample shapes. From left to right: stair step cube, where the yellow steps are scanned with different parameters; surrounded cube, where the green horse-shoe region can inflict spatter onto the standard specimen in the centre; overhang sample (see online version for colours)
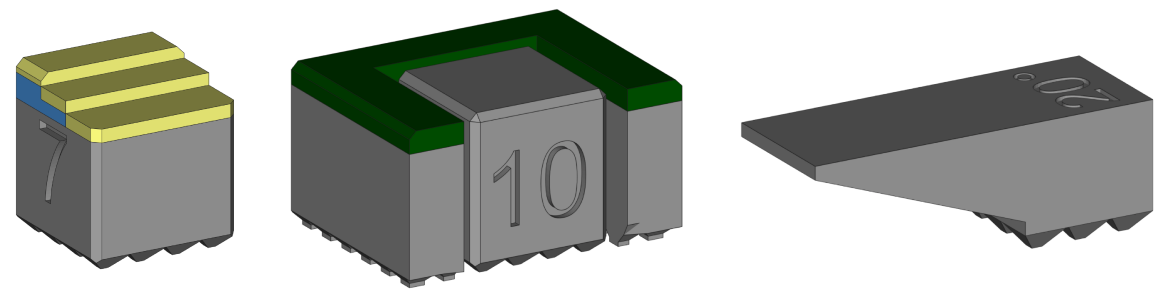

\subsection{Two-colour pyrometer}

A type H322 high-speed two-colour pyrometer manufactured by Sensortherm GmbH is used. It is based on two InGaAs photodiodes sensitive from $1450 \mathrm{~nm}$ to $1650 \mathrm{~nm}$ and $1650 \mathrm{~nm}$ to $1800 \mathrm{~nm}$ respectively. This allows for a measuring range of $700-2300^{\circ} \mathrm{C}$. Its integration is realised via a dedicated monitoring-port in the scanner (see Figure 2, $D_{1}$ ). This port extracts light in a broad spectrum (except for the laser wavelength) from the laser beam path and directs it onto a second beam-splitter $\left(D_{2}\right)$ which is employed for separating the pyrometer's corresponding band from other light (see Figure 2). By this on-axis integration, the pyrometers field of view (FOV), which is between $3-4 \mathrm{~mm}$ in diameter, constantly follows the laser-material interaction zone. The precise coaxial alignment of the pyrometer to the working laser is accomplished using a portable temperature source $\left(1200^{\circ} \mathrm{C}, \varnothing 0.3 \mathrm{~mm}\right.$ aperture). It is placed under the scanner to emulate a melt pool and aligned to the working laser. Maximising the signal on both photodiodes of the pyrometer by panning its optics indicates that they, and hence the pyrometer, are centred on the heat source and thus also on the laser. For the pyrometer to provide absolute temperature values for non-grey bodies, the emissivity slope $\left(\varepsilon_{1} / \varepsilon_{2}\right)$ for the material in question would need to be determined. But since the results from this investigation are independent of the absolute temperature values, the emissivity slope was merely guessed to obtain reasonable centigrade-like values, which are henceforth expressed in arbitrary unites (au) nevertheless. The pyrometer's serial interface is connected to a computer's USB port to record the temperature data. Due to the limited bandwidth of the serial communication, the three temperature signals $\left(T_{R}, T_{1}\right.$ and $\left.T_{2}\right)$ were merely collected at $2.5 \mathrm{kHz}$. 
Figure 2 Optical layout of the scanner. The solid red line depicts the processing beam, originating from a fibre laser $L$. The dichroitic element $D_{1}$ extracts the process signal travelling back through the scanner (green dashes). A second dichroite $D_{2}$ divides it onto the camera $C$ and the pyrometer's fibre optics $P$ (see online version for colours)

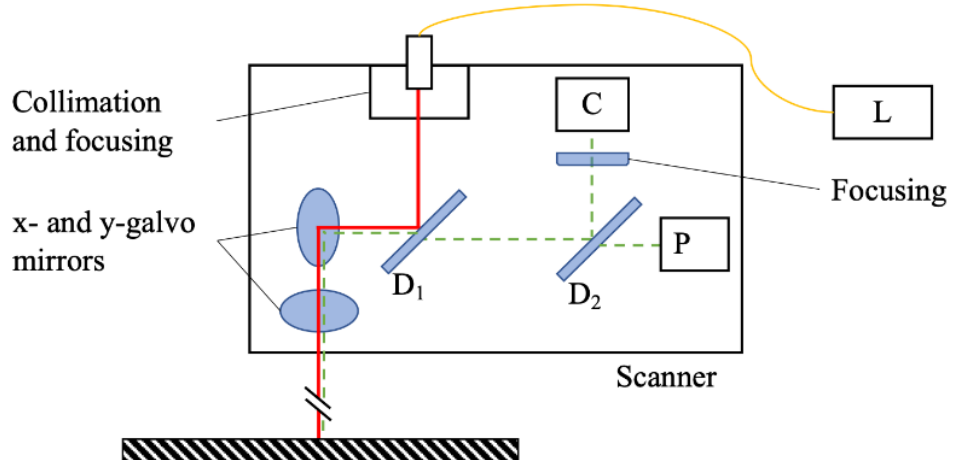

\subsection{High-speed camera}

The second channel of the scanner's monitoring port features an additional focusing unit (see Figure 2). Especially for cameras, this brings the advantage of providing focused images even when the scanner deflects the beam to the corner of the build envelope. For this study, a monochromatic CMOS camera of type CP90-3-M from Optronis $\mathrm{GmbH}$ is attached. It is connected via CoaXPress to a frame grabber. The acquisition of the $128 \times 128 \mathrm{px}$ image with 8-bit depth is set to $12.5 \mathrm{kHz}$, with an exposure time of $79 \mu \mathrm{s}$. Due to the selection of filters and the dichroic mirrors $D_{1}$ and $D_{2}$, the camera receives light mainly around $850 \mathrm{~nm}$. By beforehand placing an etched grid into the field of view and illuminating it with $850 \mathrm{~nm}$ LEDs, the resolution of the camera can be determined. In the centre of the scan field, where the following investigations take place, it is found to be $6 \mu \mathrm{m} / \mathrm{px}$. For quantitative image analysis of the blob-like signal obtained during process (see Figure 3, left), it is necessary to determine a melt grey value.

Figure 3 Illustration of the thresholding performed prior to the blob analysis. On the left, the unaltered image including blooming and noise. In the middle the thresholded image which is used for the analysis. On the right, a colour map was applied to the thresholded image to better illustrate the otherwise grey-scale image contents (see online version for colours)
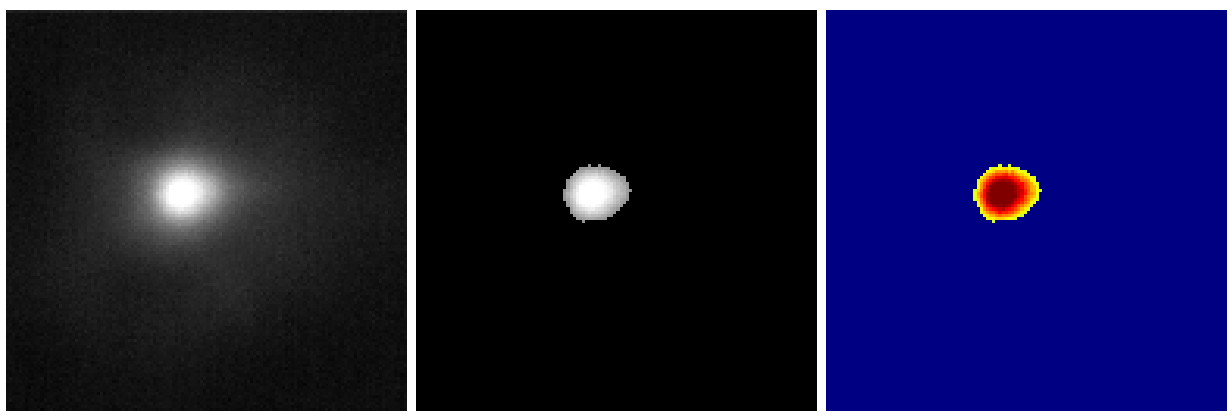
Essentially, this melt grey value is a threshold below which pixels are disregarded as blooming or noise, whereas above, pixels are counted as part of the melt pool or relevant spatter. The procedure is performed according to Craeghs et al. (2011). In essence, a line scan is generated, of which its resulting melt track width is determined using microscopy. Combined with the knowledge about the camera's resolution, the images which were acquired during the line scan are analysed in the following procedure: The threshold value is set such that the recognised illuminated field (blob) has the same diameter as the scan track width. This was done several times with varying amounts of powder. Afterwards, their average was calculated: The result was a threshold bit value of 145 (see Figure 3, middle), which was very consistent regardless the amount of powder.

\section{Signal discussion}

In the following, various process scenarios are investigated not primarily by means of the designated ratio temperature, but the single channel signals, in order to determine their relevance. At first, an overhang scenario is examined. Afterwards, the principles of twocolour pyrometry are discussed, before observing further process scenarios and in closing revisiting the overhang problem.

\subsection{Overhang scenario - negative example}

The stimulus for this research originates from findings of prior investigations (Gutknecht et al., 2020): When manually reducing the shield gas flow speed, the so-called measurement error share of the pyrometer is increased. This measurement error share is an indication that the general assumption of a grey body is violated, or the measurement is hampered elsewise; it will be discussed in the following section in more detail. Similarly, when scanning overhang regions, counterintuitive pyrometer signal development was uncovered (Chivel and Smurov, 2011; Gutknecht et al., 2021; Hooper, 2018). The overhang is chosen as an example for this paper, since it is simpler to replicate this scenario reliably. Figure 4 depicts the sensor signals from scanning towards an overhang. Scanning is performed bi-directional, and the vectors are aligned parallel to the outer $35^{\circ}$ overhang edge which is orthogonal to the etched specimen plane in Figure 5. The vector scanning order is always from solid to the overhang. Thus, the impact of the overhang increases in a discrete manner from vector to vector.

For the most part of the scanning, which happens with solid material underneath, all signals are steady and repeatable (especially before $1 \mathrm{~s}$ ). Starting at around $1.1 \mathrm{~s}, T_{R}$ starts exhibiting a consistent drop. Until around $1.17 \mathrm{~s}$, the single channel signals $\left(T_{1}\right.$ and $\left.\mathrm{T}_{2}\right)$ follow this drop, but then start rising again. Additionally, also starting at around $1.1 \mathrm{~s}$, the ratio between $T_{1}$ and $T_{2}$ starts to increase, which is the reason for the continued drop of $T_{R}$, and for the last three vectors, $T_{1}$ is permanently larger than $T_{2}$. This is visible in Figure 4 by the fact, that the $T_{1}$-line is visible below the $T_{2}$ for most of the time, but towards the end, more and more cases are present where $T_{1}$ is above $T_{2}$.

But Figure 4 holds additional information. On the secondary ordinate, the blob area coming from the camera image analysis is indicated. The signal is compliant with the pyrometer data in terms of the timing: Starting from roughly $1.15 \mathrm{~s}$, the measured 
blob area loses its consistency and experiences a decreasing trend. Similar to the single channels, a signal increase is visible for the last vectors (with exception of the very last one).

Figure 4 Plot of pyrometer and camera signal over time for an overhang geometry. Each vertical drop represents the jump between two vectors (see online version for colours)

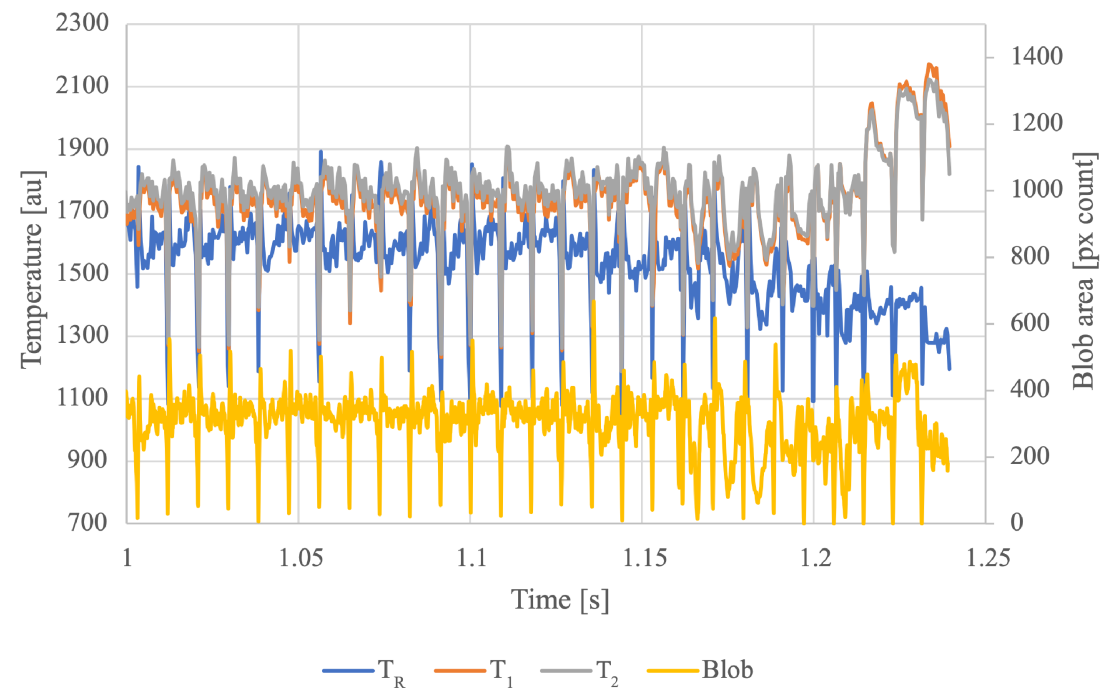

Figure 5 Overhang specimen $35^{\circ}$, vertical cut. All vectors are oriented orthogonal to the cutting plane and thus parallel to the overhang. Scan order is always from left to right, so from solid material into the overhang. The top vectors contained in the blue box are the ones plotted in Figure 4 (see online version for colours)

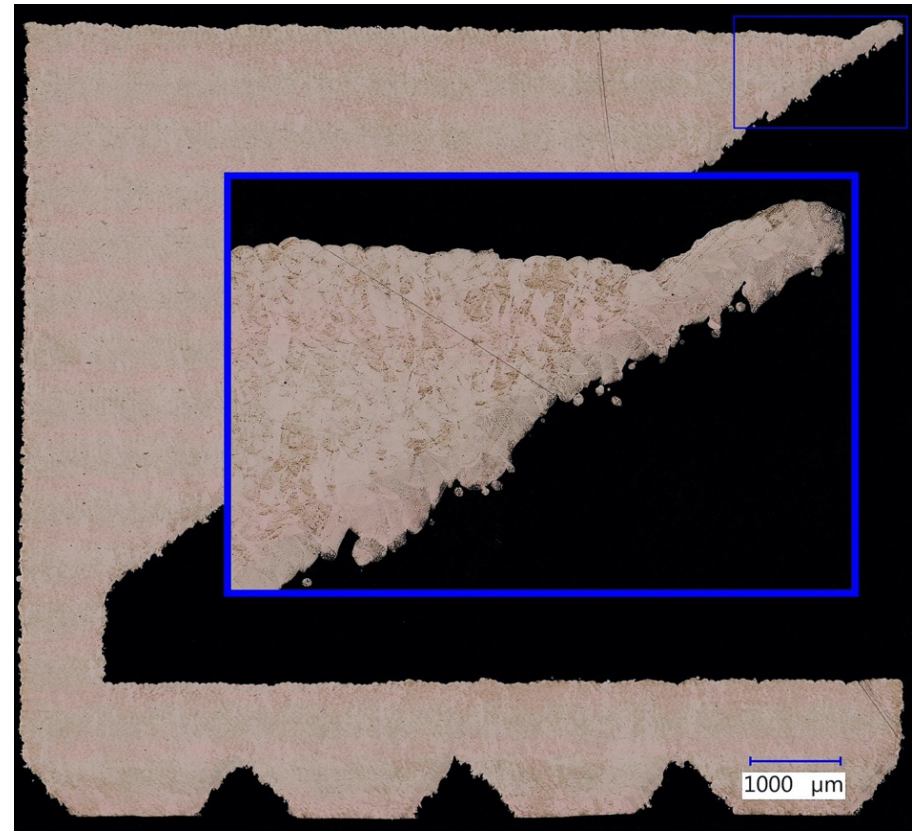


Figure 5 presents the etched specimen in a vertical cut orthogonal to the overhang edge. Most prominently, an elevated edge phenomenon is visible at the tip of the overhang. Moreover, dross formation all along the down facing side is apparent, in combination with increased melt pool size in this area. This was also qualitatively observable from the original specimen before mounting, where the down facing side showed increased surface roughness and temper colours as a general sign of too much laser power; a result from the overhang acting as a limited heat sink. Hence there is some accordance between the specimen in Figure 5, the course of the single channel signals and the blob size.

While the observations on the specimen's cut and underside (too much energy/heat) are opposed by the ratio temperature, which indicates a melt pool surface temperature decrease towards the edge. This potential misinformation can certainly be devastating when considering the ratio temperature for closed-loop control of the laser power (see Section 3.4). In the following section, the potential mechanisms behind this are discussed.

\subsection{Underlying pyrometer theory}

A two-colour pyrometer determines the ratio temperature $T_{R}$ by taking the ratio $R$ of two separate photodetector channel signals $\left(T_{1}\right.$ and $\left.T_{2}\right)$ with their individual wavelengths $\lambda_{i}$ (practically, these are not discrete wavelengths but bands). By that, the usually unknown emissivities - associated with the temperature, the wavelength and especially the material and its surface state - cancel out. At least they do so if the measured surface in question follows the grey-body assumption; the emissivities $\varepsilon_{i}$ of the two wavelengths being equal. Müller and Renz (2001) describe this principle in more detail. If the two emissivities are non-equal but remain constant in the measurement range, they can be considered as the emissivity ratio (also called emissivity slope) in the equation, which in turn still provides a sound measurement once that ratio is determined. Additionally, sensor specific tuning constants $K_{i}$ and Planck's second radiation constant $c_{2}$ are required:

$$
T_{R}=\left(\frac{\ln R-\ln \varepsilon_{1} / \varepsilon_{2}-\ln K_{1} \lambda_{2}^{5} / K_{2} \lambda_{1}^{5}}{c_{2}\left(\lambda_{2}^{-1}-\lambda_{1}^{-1}\right)}\right)^{-1}
$$

For a successful measurement, there are some opposing factors needing to be considered: Generally, a trade-off needs to be made when selecting the wavelength bands. On the one hand, the bands need to be close to each other for them to have the same emissivity and exhibit the same behaviour regarding potential emissivity changes. On the other hand, they need to be far enough apart, such that the ratio of their signals still yields significant results and is not dominated by noise. Additionally, having wavelengths far apart increases the possibility of selective attenuation, since due to the distance of the separate bands, it is more likely that one is attenuated independently of the other. On the contrary: should selective attenuation occur, the closeness of two neighbouring bands is adverse since the ratio $R$ is inherently closer to 1 and thus more susceptible to changes/noise resulting from said attenuation.

A change in the signal ratio $R$ can have two main causes:

i The emission is attenuated selectively, which could occur for example with the presence of soot in the beam path or condensate on the laser window. 
ii The amount of emitted radiation changes:

a This is either because the temperature changes, which is the desired cause since it is the intended measuring principle,

b or due to the emissivity itself changing differently on the two channels, i.e., for the two wavelength bands. This can be considered a measurement error, as such changes usually happen unnoticed and are thus not accounted for in the above equation.

The two wavelength bands of the pyrometer in question here are delimited in the radiation plot depicted in Figure 6 with vertical lines. It becomes apparent, that at least for an ideal black body and for temperatures above $1800 \mathrm{~K}$, channel 2 is expected to receive more radiation than channel 1 . Indeed, according to Wien's displacement law, the pyrometer's switching wavelength (dashed line) of $1650 \mathrm{~nm}$ coincides with the peak of the spectral radiance for a black body temperature of $1756 \mathrm{~K}$. Which happens to be slightly above the melting temperature of the given alloy.

Figure 6 Plot of the black body radiation between $0 \mu \mathrm{m}$ and $3 \mu \mathrm{m}$ for the relevant temperature range of the pyrometer. The upper and lower cut-off thresholds K1 and K2 delimiting the spectral bands of the associated photodiodes (see online version for colours)

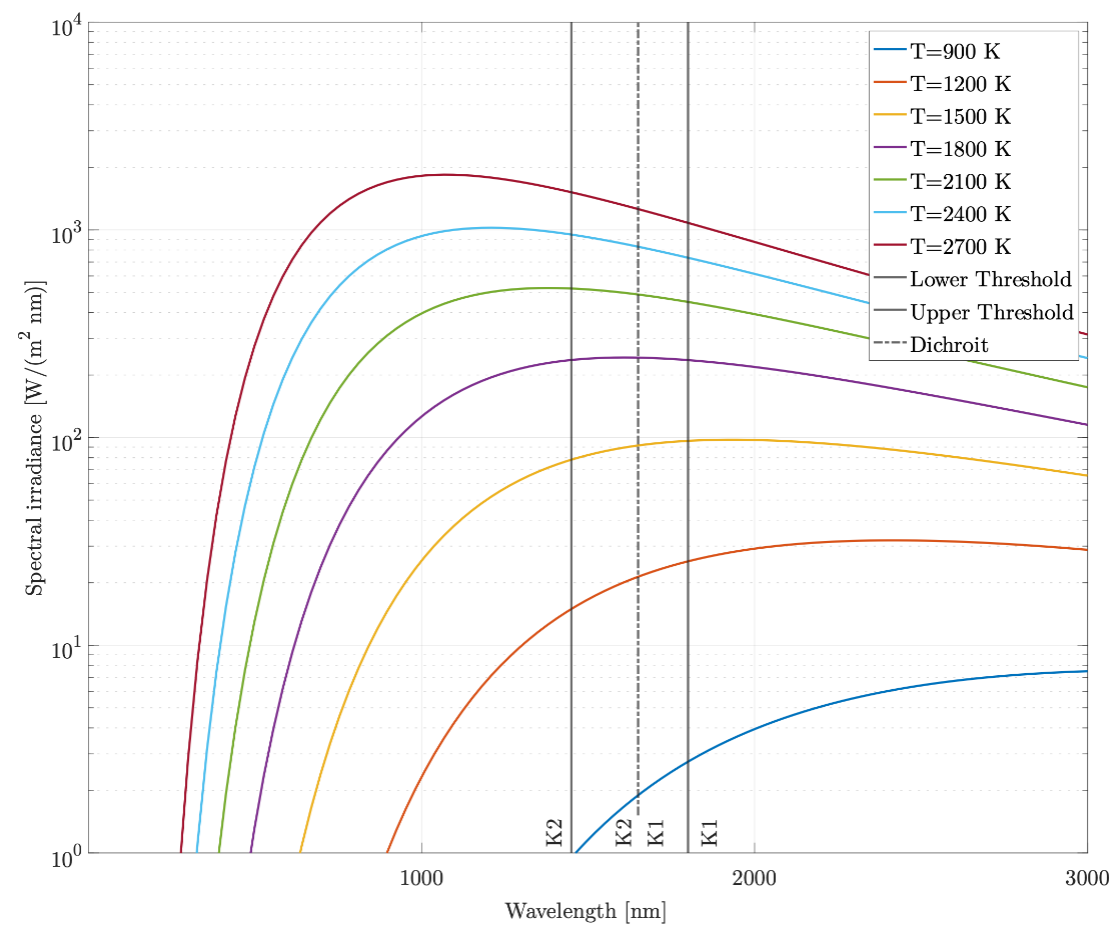

During calibration though, the individual amplifications of the single channels are intentionally set such, that $T_{2}>T_{1}$ for the whole measuring range between $700^{\circ} \mathrm{C}$ and $2300^{\circ} \mathrm{C}$ (the broader bandwidth of channel 2 also helps with this, see Figure 6 again)! The effectiveness can be demonstrated by stationary pointing the laser beam onto a solid 
plate of steel and recording the pyrometer values during the heat-up process, which is plotted in Figure 7.

Figure 7 Stationary laser beam on steel plate. The discrepancy between the single channel signals and the ratio temperature originates from that fact, that the resulting stationary melt pool is smaller in size than the surrogate heat source used for the initial calibration (see online version for colours)

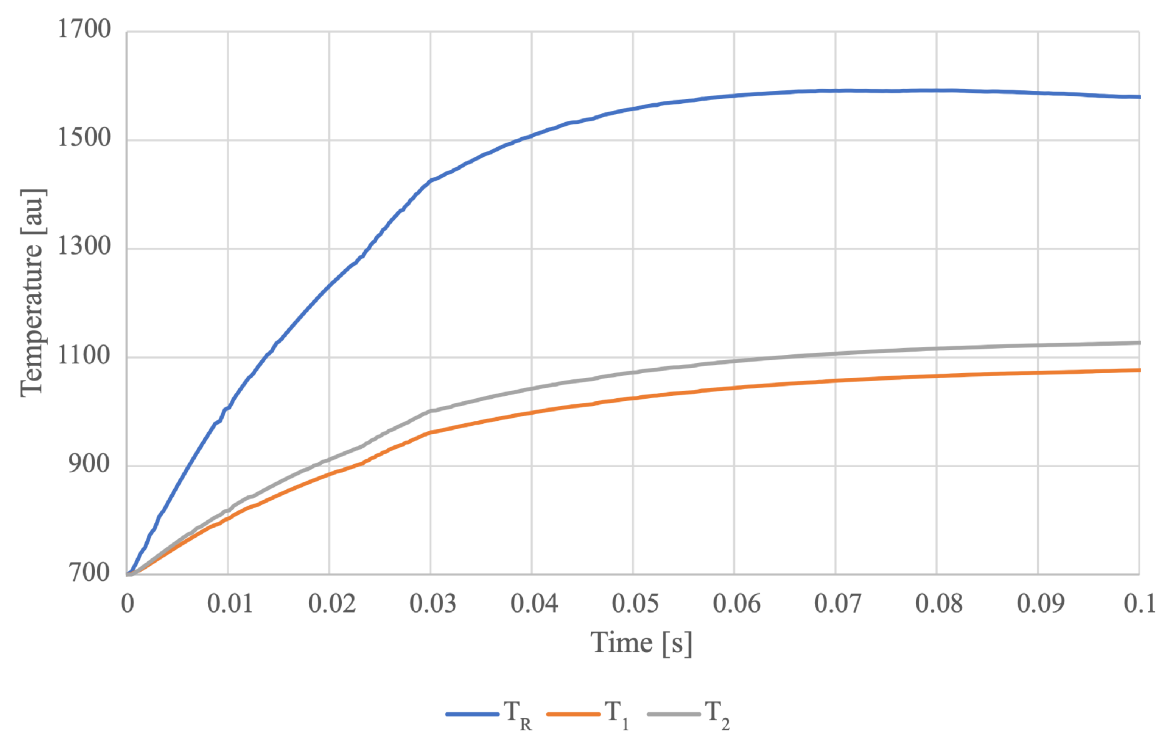

By this setup, an inversion in the single channel ratio $\left(T_{2}>T_{1} \rightarrow T_{1}>T_{2}\right)$, as it was demonstrated with the last three scan vectors of Figure 4, is indicative of an attenuation phenomenon or any emissivity change (i or ii(b)). Furthermore, it makes the pyrometer very robust regarding the temperature as the sole cause of the inversion (ii(a)). This robustness applies at least as long as the melt pool remains in a commensurate size further discussion below.

Figure 8 depicts a selection of different melt pool states with their isotherms, where:

a Stands for 'normal' process conditions in conduction mode welding.

b Illustrates the appearance of a large spatter or powder particle, temporarily reducing the overall temperature as it withdraws heat to attain the same temperature level as the melt pool.

c Shows a scenario, where the heat conductivity is impaired (for example in an overhang situation).

d Depicts a keyhole mode, which potentially exhibits different emission states due to the increased presence of vapourisation or experiences selective attenuation because of that vapour.

Both (b) and (c) highlight the possibility for the portion of cooler melt pool to become significantly larger than anticipated/calibrated. Such a drastic shift in emission can also result in the above-described channel inversion, since the asymmetric amplification of the channels is overruled at some point. 
Figure 8 A selection of different melt pool states in top-view with the colours as arbitrary isotherms: (a) conduction mode melt pool; (b) conduction mode melt pool confronted with a large particle; (c) situation with bad heat conductivity underneath and (d) keyhole regime. Pyrometer field of view (FOV) not to scale (see online version for colours)

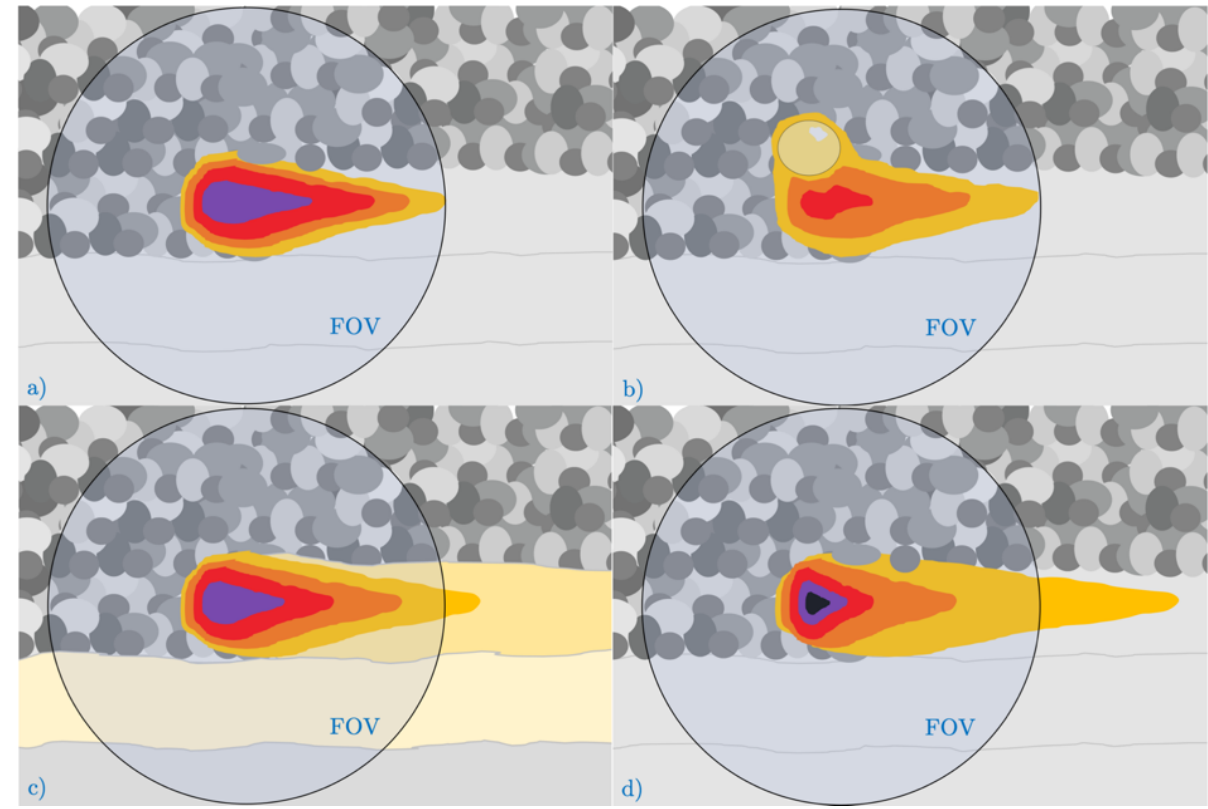

Therefore, since not all potential scenarios leading to the inversion of the channels can be considered a measuring error (which is manifested in the following, alongside the fact that all occurrences are relevant nonetheless), the neutral terminology channel inversion is used instead of measuring error.

\subsection{Application of channel inversion monitoring}

To investigate the effectiveness of the channel inversion as an indicator for process anomalies, a series of provoked and unhealthy process conditions are examined hereinafter. Each experiment was conducted across several layers to ensure a stationary condition. During this condition, one representative layer was recorded. Layers themselves comprise up to 140 vectors and hence still constitute a large enough sample for statistical significance. The signals are being averaged per layer to compress them into one number and compare them with other process conditions and a reference value, as presented in the following tables. The reference value is taken of each same part from a layer where standard parameters are applied. For evaluating the representativeness of such a layer over the course of a build job, in Table 3 two reference layers are presented. One from the lower region of the part and one from the upper region. Their values are almost identical.

Uneven powder layers and unnaturally large particles (e.g., deposits of spatter) can be reasons for stochastic process deviations. To simulate this, a standard specimen is surrounded by a horse-shoe shaped auxiliary body with a lateral distance of only $1 \mathrm{~mm}$ (see Figure 1). The latter is configured such, that it is scanned prior to the standard 
specimen. Most importantly, its laser power level is deliberately altered (500 instead of $200 \mathrm{~W})$ to cause increased spatter ejection. As a result of the proximity, this spatter is then deposited onto the powder layer of the to-be-scanned standard specimen. To not completely jeopardise the process, this is performed for a limited number of layers only. And indeed, the standard specimen's local relative density of the layer region with increased spatter is determined to be $99.745 \%$, while the density of unimpaired regions of the same sample is measured as $99.994 \%$ using metallographic images for pore detection and quantification (specifics at the end of this section). The essence of the sensors' melt pool monitoring data from that spatter inflicted specimen is summarised in Table 2 for three representative layers. The reference layer is unaffected by the spatter. The spatter layer is the first among several layers where the neighbouring part's laser power is increased and spatter deposition is happening, and the post-spatter layer is recorded five layers after the auxiliary part has finished and no more neighbouring spatter is generated.

Table 2 Layer average sensor values of the specimen subjected to spatter of a neighbouring body

\begin{tabular}{cccccccc}
\hline & Layer & Inversions & \multicolumn{3}{c}{ Temperature [au] } & \multicolumn{2}{c}{ Blob [au] } \\
\hline № & Type & {$[\%]$} & $T_{R}$ & $T_{1}$ & $T_{2}$ & Intensity & Area \\
\hline 41 & Reference & 1.22 & 1555 & 1723 & 1747 & 197 & 333 \\
317 & Spatter & 12.88 & 1487 & 1780 & 1794 & 195 & 325 \\
341 & Post-spatter & 0.81 & 1566 & 1718 & 1743 & 196 & 328 \\
\hline
\end{tabular}

A layer mildly (according to the local density) affected by spatter deposition exhibits already a tenfold increase in pyrometer single channel inversion occurrence. In parallel, the images from the camera (blob area) slightly point towards a smaller melt pool, visible at $850 \mathrm{~nm}$. This generally agrees with the illustration Figure $8(\mathrm{~b})$ ), where the melting of a large spatter particle for one thing increases the overall area of the melt pool, but also reduces the overall temperature level. Since the short wavelength associated with the camera is generally governed by higher temperatures, it ultimately captures a smaller blob. While the single channels of the pyrometer receive a signal increase due to the increased amount of radiation at their respective wavelength band coming from the larger melt pool.

As demonstrated in Table 3, similar results are also obtained when manually changing the layer height (see the stair-step specimen in Figure 1). For the $90 \mu \mathrm{m}$ case, the camera more prominently notices a change in the melt pool, which is reflected by the image analysis as a smaller blob. The specimens' density is affected again, for obvious reasons: the parameter set was developed for a layer thickness of $30 \mu \mathrm{m}$.

Table 3 Layer average sensor values of the specimen subjected to varying layer heights

\begin{tabular}{cccccccccc}
\hline & Layer & Inversions & \multicolumn{4}{c}{ Temperature $[\mathrm{au}]$} & \multicolumn{2}{c}{ Blob [au] } & \multicolumn{2}{c}{ Density } \\
\hline № & Height $[\mu \mathrm{m}]$ & {$[\%]$} & $T_{R}$ & $T_{I}$ & $T_{2}$ & Intensity & Area & {$[\%]$} \\
\hline 42 & 30 & 0.48 & 1604 & 1681 & 1712 & 195 & 328 & 99.990 \\
312 & 30 & 0.66 & 1606 & 1682 & 1713 & 196 & 327 & 99.994 \\
339 & 60 & 2.77 & 1538 & 1763 & 1784 & 196 & 332 & 99.968 \\
364 & 90 & 32.46 & 1438 & 1831 & 1836 & 191 & 277 & 98.361 \\
\hline
\end{tabular}


In both cases, the indication given by $T_{R}$ is correct, as for the increased layer height, additional laser power would be required to omit lack-of-fusion (LOF), which the etched specimen uncovers in Figure 9. Even though the ratio temperature is correct for this phenomenon, the channel inversion is nevertheless a good indicator with a high sensitivity.

Figure 9 Region of the specimen where the layer height is increased to $90 \mu \mathrm{m}$. Lack-of-fusion voids are clearly visible (see online version for colours)

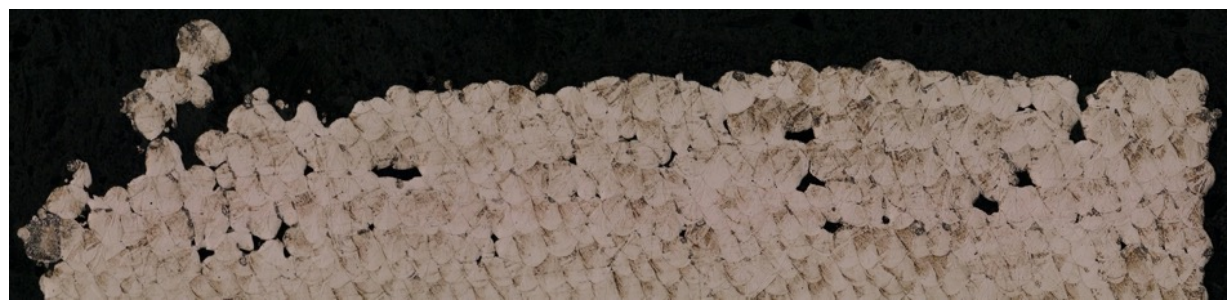

A defocused laser beam is emulated using the scanner's zoom functionality to widen the beam waist. This will result in a shallower but wider melt pool, which in turn can result in LOF porosity. This is again reflected in the channel inversions, as Table 4 demonstrates. In this case, the blob area and intensity are reduced likewise and more notably.

Table 4 Layer average sensor values of the specimen subjected to varying spot diameters

\begin{tabular}{cccccccccc}
\hline \multicolumn{2}{c}{ Layer } & Inversions & \multicolumn{4}{c}{ Temperature $[\mathrm{au}]$} & \multicolumn{2}{c}{ Blob [au] } & \multicolumn{2}{c}{ Density } \\
\hline № & Spot $[\mu \mathrm{m}]$ & {$[\%]$} & $T_{R}$ & $T_{1}$ & $T_{2}$ & Intensity & Area & {$[\%]$} \\
\hline 42 & 86 & 0.71 & 1611 & 1678 & 1710 & 194 & 324 & 99.986 \\
312 & 120 & 1.58 & 1551 & 1698 & 1724 & 174 & 221 & 100.000 \\
339 & 150 & 2.59 & 1507 & 1757 & 1774 & 159 & 160 & 99.717 \\
364 & 180 & 7.79 & 1476 & 1754 & 1768 & 114 & 90 & 99.582 \\
\hline
\end{tabular}

Although not as sensitive as in the applications afore, the channel inversion still indicates a problem. The ratio temperature again hints the correct trend, as more laser power would be required to compensate for the widened beam diameter to maintain a sufficient melt pool temperature.

All relative material densities are determined by the pre-etched images of the samples. Roughly $0.3 \mathrm{~mm}$ on the border of the parts are disregarded. Threshold intensity above which a pixel is counted as solid is set to 166 out of 255 bits. Furthermore, connected blobs with a size equivalent to a circle with diameter $10 \mu \mathrm{m}$ or smaller are neglected. Most of the few channel inversions encountered under normal process conditions (which is usually a number below 1\%) coincide with the laser-on and -off events on the turning points.

This chapter provided evidence, that with the correct setup, the single channel inversion of the pyrometer is a very sensitive tool for detecting process anomalies. In some cases, this also suggests as to when to consult the single channels for deciding 
whether $T_{R}$ is impaired (overhang, shield gas) or not (spatter, layer thickness, defocus). However, the root cause for the inversion is not determined yet, some ideas are discussed in Section 3.2 based on Figure 8 though.

\subsection{Overhang scenario revisited}

To further investigate the relevance of the findings presented above regarding the channel inversion highlighting a potential measurement error, the initial problem of building an overhang is revisited. A more difficult geometry with an inclination angle to the horizontal of only $20^{\circ}$ (see Figure 1) is manufactured three times with different approaches:

a open-loop, fixed laser power as a reference

b closed-loop, PID controlled laser power with $T_{R}$ as input to demonstrate the effects of the measurement error

c closed-loop, PID controlled laser power with $T_{1}$ as input to overcome the measurement error.

The results, in form of the specimens' underside, is depicted in Figure 10 and the general outcome is discussed below.

Figure $1020^{\circ}$ overhang specimens, left the open-loop specimen (but with $25 \%$ power reduction), in the middle the closed-loop $T_{R}$-based where the controller was manually overruled to not compromise the whole build job, and on the right the closed-loop $T_{1}$-based specimen (see online version for colours)

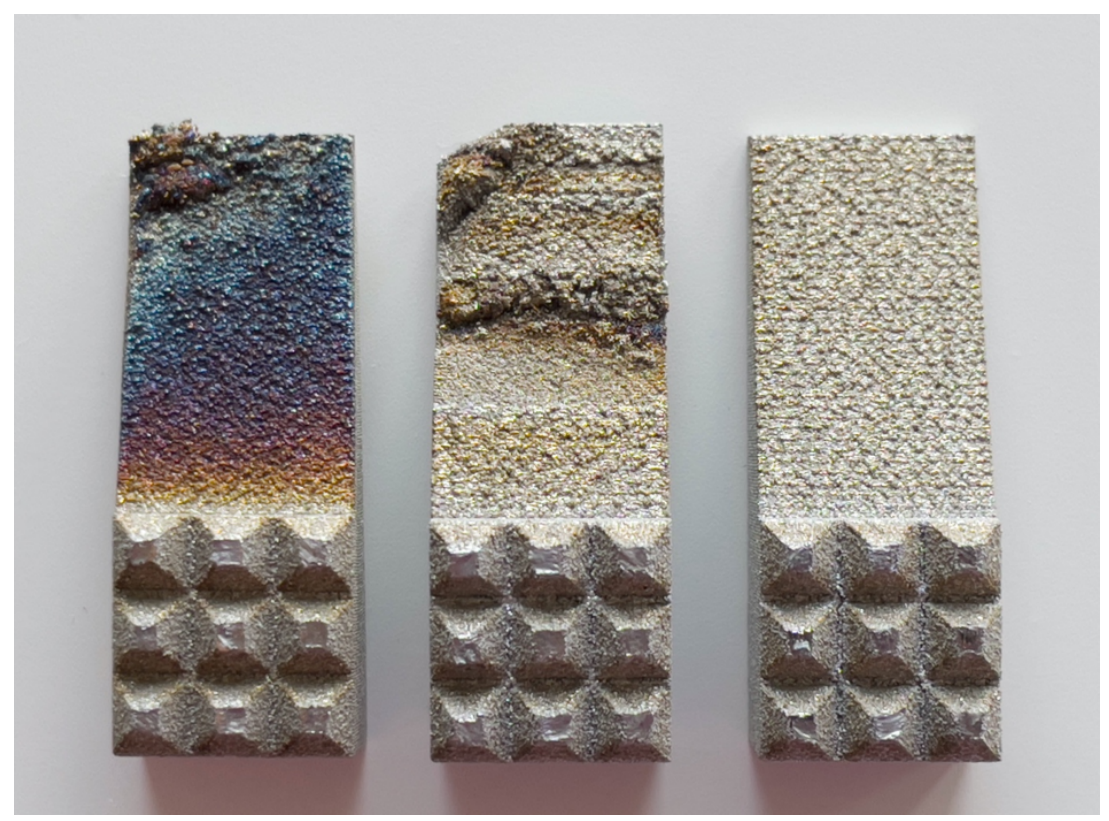

The open-loop specimen was successfully manufactured only due to the fact, that the overall laser power was reduced by $25 \%$ from the original setpoint. Nonetheless, the underside demonstrates evidence of overheating. The second specimen features heavy 
dross formation and again annealing colours, combined with some delamination. The reason for this being the PID-controller acting according to the ratio temperature, which exhibited the inverse behaviour already demonstrated in Section 3.1. Thus, every time the scanning approached the overhang, the laser power was increased when it should have been decreased, demonstrating the consequence of the measurement error. The only reason the part was successfully built at all, is the controller setpoint for the ratio temperature being manually and step-by-step reduced over the course of the build job in order to potentially attain a level where the inversion would not occur, which was never the case. As a result of that, the controller was basically overruled, as it was limited in its capabilities to increase the power. This yielded essentially the same result as the openloop approach: reduced laser setpoint, i.e., the controller was de facto inoperative. Whereas the specimen depicted on the right was manufactured with $T_{1}$ as the controller input, which was successful. The target temperature value was determined by taking the average of a standard specimen's $T_{1}$ value, no further manual interference was needed. Since the wavelength bands of both single channels are very close, their signals $T_{1}$ and $T_{2}$ exhibit the same behaviour. Therefore, both are equally suitable as an input for the controller.

\section{Conclusion and outlook}

In this study, the principles of two-colour pyrometry are investigated to determine the relevance of the single channel signals.

- It was demonstrated, that monitoring these signals in addition to the ratio temperature brings significantly more information content.

- With the right setup, a wrong ratio temperature calculated on the usual assumptions of two-colour pyrometry can easily be uncovered.

- Additionally, benefits were demonstrated for the closed-loop control approaches in manufacturing an overhang scenario.

- However, although the overhang was successfully manufactured using a single channel signal only, this does not deem the two-colour approach superfluous.

For further improvements and robustness, the ratio temperature needs to be taken into account primarily. Switching to the single channel should be considered as the ultimo ratio, in case of a channel inversion for example.

Open questions remain on the topic of the transferability towards different materials, which might exhibit different emissivity behaviours. Additionally, the applicability for high-power parameters needs to be checked, as the given findings all revolve around a moderate $200 \mathrm{~W}$ parameter set. Furthermore, for robust industrial application, a sophisticated algorithm needs to be implemented, which can switch between the singlechannels and the ratio temperature as the controller input and provide general interpretation as to what is the root cause for a channel inversion. Specifically, a distinction for when the channel inversion indicates a measurement error on the ratio temperature needs to be elaborated. Finally, adjustments to the ratio/calibration could potentially be improved to increase the sensitivity and prevent wrong indications. 


\section{References}

Chivel, Y. and Smurov, I. (2011) 'Temperature monitoring and overhang layers problem', Physics Procedia, Vol. 12, pp.691-696.

Colosimo, B.M. and Grasso, M. (2020) 'In-situ monitoring in L-PBF: opportunities and challenges', Procedia CIRP, Elsevier, B.V., pp.388-391.

Craeghs, T., Clijsters, S., Yasa, E., Bechmann, F., Berumen, S. and Kruth, J-P. (2011) 'Determination of geometrical factors in layerwise laser melting using optical process monitoring', Optics and Lasers in Engineering, Vol. 49, pp.1440-1446.

Everton, S.K., Hirsch, M., Stravroulakis, P., Leach, R.K. and Clare, A.T. (2016) 'Review of in-situ process monitoring and in-situ metrology for metal additive manufacturing', Materials and Design, Vol. 95, pp.431-445.

Forien, J.B., Calta, N.P., Depond, P.J., Guss, G.M., Roehling, T.T. and Matthews, M.J. (2020) 'Detecting keyhole pore defects and monitoring process signatures during laser powder bed fusion: a correlation between in situ pyrometry and ex situ X-ray radiography', Additive Manufacturing, Vol. 35, p.101336.

Gutknecht, K., Haferkamp, L., Cloots, M. and Wegener, K. (2020) 'Determining process stability of laser powder bed fusion using pyrometry', Procedia CIRP, Vol. 95, pp.127-132.

Gutknecht, K., Cloots, M., Sommerhuber, R. and Wegener, K. (2021) 'Mutual comparison of acoustic, pyrometric and thermographic laser powder bed fusion monitoring', Materials \& Design, Vol. 210, p.110036.

Hagedorn, Y. and Pastors, F. (2018) 'Process monitoring of laser beam melting', Laser Technik Journal, pp.54-57.

Hooper, P.A. (2018) 'Melt pool temperature and cooling rates in laser powder bed fusion', Additive Manufacturing, Vol. 22, pp.548-559.

Jurrens, K. (2013) Measurement Science Roadmap for Metal-Based Additive Manufacturing, National Institute of Standards and Technology.

Lu, Q.Y. and Wong, C.H. (2018) 'Additive manufacturing process monitoring and control by nondestructive testing techniques: challenges and in-process monitoring', Virtual and Physical Prototyping, Vol. 13, pp.39-48.

Mani, M., Lane, B.M., Donmez, M.A., Feng, S.C., Moylan, S. and Fesperman, R. (2015) Measurement Science Needs for Real-Time Control of Additive Manufacturing Powder Bed Fusion Processes, NIST Interagency/Internal Report (NISTIR).

Mani, M., Lane, B.M., Donmez, M.A., Feng, S.C. and Moylan, S.P. (2017) 'A review on measurement science needs for real-time control of additive manufacturing metal powder bed fusion processes', International Journal of Production Research, Vol. 55, pp.1400-1418.

Mitchell, J.A., Ivanoff, T.A., Dagel, D., Madison, J.D. and Jared, B. (2020) 'Linking pyrometry to porosity in additively manufactured metals', Additive Manufacturing, Vol. 31, p.100946.

Müller, B. and Renz, U. (2001) 'Development of a fast fiber-optic two-color pyrometer for the temperature measurement of surfaces with varying emissivities', Review of Scientific Instruments, Vol. 72, pp.3366-3374.

Pavlov, M., Doubenskaia, M. and Smurov, I. (2010) 'Pyrometric analysis of thermal processes in SLM technology', Physics Procedia, pp.523-531.

Purtonen, T., Kalliosaari, A. and Salminen, A. (2014) 'Monitoring and adaptive control of laser processes', Physics Procedia, Vol. 56, pp.1218-1231.

Quarshie, R., MacLachlan, S., Reeves, P., Whittaker, D. and Blake, R. (2012) Shaping our National Competency in Additive Manufacturing, Additive Manufacturing, Special Interest Group.

Renken, V., von Freyberg, A., Schünemann, K., Pastors, F. and Fischer, A. (2019) 'In-process closed-loop control for stabilising the melt pool temperature in selective laser melting', Progress in Additive Manufacturing, Vol. 4, pp.411-421. 
Spears, T.G. and Gold, S.A. (2016) 'In-process sensing in selective laser melting (SLM) additive manufacturing', Integrating Materials and Manufacturing Innovation, Vol. 5, pp.1-25.

Tapia, G. and Elwany, A. (2014) 'A review on process monitoring and control in metal-based additive manufacturing', Journal of Manufacturing Science and Engineering, Transactions of the ASME, p.136.

Thombansen, U., Gatej, A. and Pereira, M. (2014) 'Process observation in fiber laser-based selective laser melting', Optical Engineering, p.54.

Zhang, Y., Fuh, J.Y.H., Ye, D. and Hong, G.S. (2019) 'In-situ monitoring of laser-based PBF via off-axis vision and image processing approaches', Additive Manufacturing, Vol. 25, pp.263-274. 